\title{
DCT APPROXIMATION FOR LOW BIT RATE CODING USING A CONDITIONAL TRANSFORM
}

\author{
Ricardo L. de Queiroz \\ Xerox Corporation, 800 Phillips Rd., 128-27E, Webster, NY, 14580 \\ queiroz@ieee.org
}

\begin{abstract}
A transform approximation is explored for speeding up the software compression of images and video. It is used to replace the regular DCT whenever only few DCT coefficients are actually encoded. A conditional transform is proposed to only transform the data that it determines to be relevant. The approximation is applicable to environments combining the requirements of high compression and low complexity.
\end{abstract}

\section{INTRODUCTION}

Image transmission is commonplace. Among numerous applications, the ITU has recently standardized a system for color fax [1]. In its baseline mode, color fax operates with a handshaking procedure similar to that of black and white fax. After connection is established, the scanned image is JPEG [2] compressed and transmitted. Often, the compression ratios are quite large. Other facsimile systems such as Internet Fax are being devised [1]. Video is also pervasive on the internet and MPEG compression [3] is a popular video representation format.

We are concerned with systems where the image is input or generated on-the-fly and transmitted immediately. This is the case in live transmission of video and stills. In color fax, image parameters are only determined after handshaking. Hence, as is the case of live production of stills and video, compression has also to be performed in real time. Compression standards such as JPEG [2] or MPEG [3] rely on sequential transform, quantization, and entropy coding. Typically, all image data is transformed and quantization is applied to every transformed coefficient. These two steps typically drain a significant fraction of the compression computation and are independent of the image data or of the compression target.

In summary, we are concerned with systems in which software-based compression is performed on-the-fly and where it is desirable to reduce computational complexity. Our goal is to speed up the compression by replacing the real transformation by a low cost approximation. We examine the case of the discrete cosine transform (DCT) and concentrate on the JPEG image coding standard, although results would also apply to MPEG, or, effectively, to any block transform based compression system. A companion paper discusses reduced transforms and fast image analyzers [4].

\section{CONDITIONAL REFINEMENT}

If we can determine if there are no non-zero high frequency coefficients in certain blocks, we can approximated a reduced DCT, which just implements the DCT for the low frequency coefficients. This concept has been applied in [5]-[9]. In-

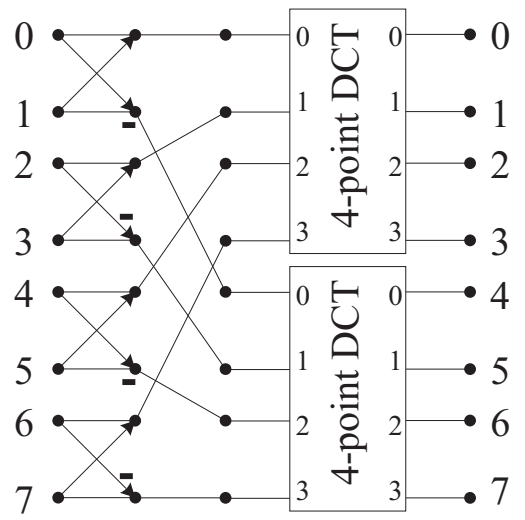

Figure 1. Approximation of an 8-point DCT.

variably, one uses a reduced-complexity DCT approximation, which is supposed to be only used when it is estimated that there is no high frequency content on the block. The problem is the estimation step. It must be reliable enough not to cause artifacts and fast enough not to offset the savings of a fast DCT approximation. As a starting point, we have developed an approximation to the DCT, depicted in Fig. 1 for an 8-channel transform, which is similar to the "subband-DCT" scheme [7]. It is also similar to the approximation in [6], except for one small but important change to the last stage. The 8-point DCT is approximated by 4 simple Haar transforms (sum and difference) followed by DCTs. The output of the sum is a simple low-pass version of the block samples, while the output of the difference is like a high pass signal. Now, each resulting 4-point signal (low- and high-pass) is transformed by a 4-point DCT to get an 8-point transformed sequence. DCT bases are compared to the bases of the approximation in Fig. 2.

The advantage of using such an approach is because after the Haar transforms, the low- and the high-frequency coefficients have already been separated. Since the DCT is orthogonal, the signal power before and after the DCT-4 is the same, so that we can estimate the signal power of the transformed coefficients just by estimating the power of the Haar-transformed samples.

The proposed system is illustrated in Fig. 3. Using the Haar transform the image is divided into 4 quadrants: sum or difference in both directions, and the other two combinations. The low-pass quadrant is transformed by the $4 \times 4$ DCT. Each of the 3 other quadrants is checked for activity. This is done by comparing each sample to a threshold. If any sample is above the threshold, the DCT for that section is computed, otherwise the DCT coefficients in that region are 

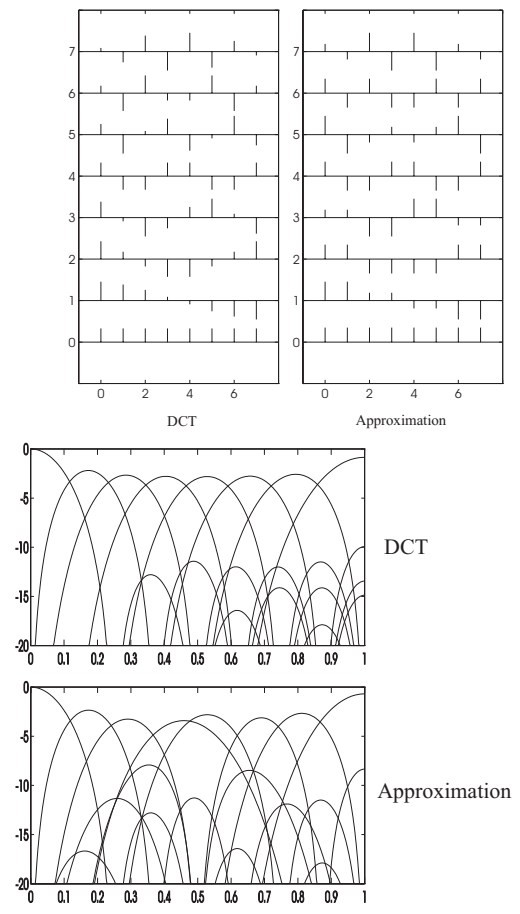

Figure 2. Comparison of bases of the DCT and of its approximation. Top: spatial domain; bottom: frequency response of the bases when viewed as filters.

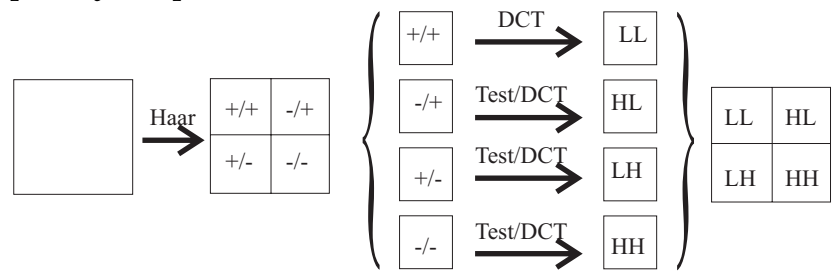

Figure 3. Conditional transform proposed here. High-pass segments of the DCT coefficients are only computed if samples before the DCT-4 are deemed relevant.

directly set to zero. The threshold can be set as the average of the quantizer steps in the respective DCT region as in the example in Fig. 4. This average can be divided by 2 to dispense applying the scaling step $(\times 1 / 4)$ in the Haar averaging. When a quadrant is considered active (some sample above threshold) it is transformed using a $2 \mathrm{D} 4 \times 4 \mathrm{DCT}$, which incorporates the normalization factor from the Haar transform along with its own.

Counting additions, multiplications, compares (to zero), shifts, etc. as one operation (op), the total complexity for the $8 \times 82 \mathrm{D}$ DCT is 768 ops assuming a standard separable algorithm [10]. Using the same algorithm for the $4 \times 42 \mathrm{D}$ DCT the complexity is 112 ops. Other algorithms can implement the $8 \times 82 \mathrm{D}$ DCT with 672 ops [11] or 572 ops [12] (discarding scalings), but we will stick to the first method since the performance improvement we propose is relative. The average complexity per block for the proposed transform is $336+112 \nu$ ops, where $\nu$ is the average number of active high-frequency bands, i.e. bands above the threshold. The relative complexity compared to the DCT case is

\begin{tabular}{|rrrr|rrrr|}
\hline 6 & 8 & 10 & 13 & 21 & 35 & 62 & 117 \\
8 & 9 & 12 & 17 & 28 & 47 & 84 & 135 \\
10 & 12 & 16 & 25 & 40 & 69 & 102 & 165 \\
13 & 17 & 25 & 38 & 64 & 89 & 134 & 219 \\
\hline 21 & 28 & 40 & 64 & 86 & 123 & 189 & 255 \\
35 & 47 & 69 & 89 & 123 & 181 & 255 & 255 \\
62 & 84 & 102 & 134 & 189 & 255 & 255 & 255 \\
117 & 135 & 165 & 219 & 255 & 255 & 255 & 255 \\
\hline
\end{tabular}

\begin{tabular}{|rr|}
\hline \begin{tabular}{rr}
$X$ & 44 \\
44 & 108 \\
\hline
\end{tabular} \\
\hline Associated \\
Thresholds
\end{tabular}

Q-table
Figure 4. An example quantizer table and associated thresholds to test relevance of subbands in the conditional transform. Thresholds are half the averages of the quantizer step entries in each quadrant.

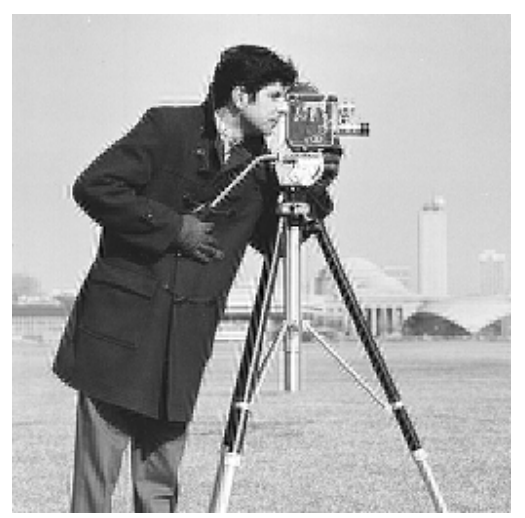

Figure 5. Transform mismatch example. Image cameraman $(256 \times 256$ pixels $)$ transformed using the conditional approximation but reconstructed using the DCT.

$\phi=0.4375+0.1458 \nu$. Hence, in the best case, the complexity is less than half of that of the DCT, while in the worst case it is at least $12.5 \%$ less that the DCT. The following table summarizes the behavior of $\nu$ and $\phi$ for image "Lena", at different compression ratios (CR), where it should be appreciated how quickly the complexity $\phi$ approaches its minimum value.

\begin{tabular}{|c|c|c|c|c|c|}
\hline CR & 4.5 & 7.7 & 11.7 & 18 & 26 \\
\hline$\nu$ & 1.80 & 0.84 & 0.36 & 0.15 & 0.02 \\
\hline$\phi$ & 0.70 & 0.56 & 0.49 & 0.46 & 0.44 \\
\hline
\end{tabular}

As in the other approximations in this paper, there is a quality reduction price one pays for the increase in speed. Let us consider two decoder scenarios. In the first, the decoder uses the inverse of the approximated transform (inverse $4 \times 4$ DCTs, and inverse Haar transforms). The compression performance is a bit below that of the DCT because the transform itself is not as close to the optimal as the DCT is for a Markov image model. The rate-distortion curves for the approximation and for the DCT are comparable, with the DCT being consistently superior (typically by $1 \mathrm{~dB}$ or so). In the second scenario, a regular inverse DCT is used in the decoder causing a transform mismatch as exemplified in Fig. 5. There are jagged ringing artifacts near sharp edges. These artifacts may or may not be noticeable in a high-resolution low-bit-depth environment, e.g. printing. In any case, if there is a potential transform mismatch it is advisable to use the conditional transform for low-bit-rate cases only. 


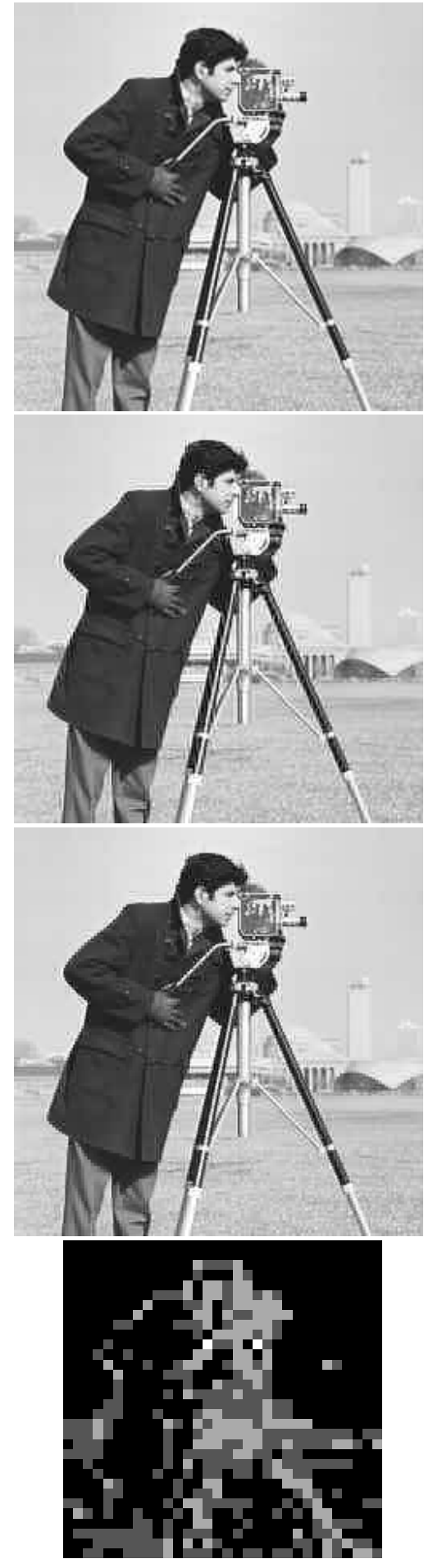

Figure 6. Image cameraman compressed at moderate bit rates using JPEG. Top left: regular DCT (0.63 bpp, PSNR = 27.7dB). Top right: approximated compression but using regular inverse DCT (0.62 bpp, PSNR = 25.4dB). Bottom left: approximated transform $(0.62 \mathrm{bpp}, \mathrm{PSNR}=26.4 \mathrm{~dB})$. Bottom right: map with number of active bands.

\section{PERFORMANCE}

Figure 6 compares decompressed images after a compression to bit rates around $0.63 \mathrm{bpp}$. It is shown the image cameraman after compression using the proposed conditional transform and decompression using both an approximated transform and using the regular inverse DCT. The image after compression using regular JPEG at a similar rate is also shown in Fig. 6, along with the map with the number of active bands in each block (black $=0$; white $=3$ ). Note the ringing artifacts around the edges of the image where the mismatch between transforms occurred. The image which used the approximation in both compression and decompression stages is comparable to the straight JPEG case. Figure 7 repeats the results from Fig. 6 for a higher compression ratio, i.e. bit rates around $0.27 \mathrm{bpp}$. In this case, all the images present similar visual reconstruction quality, despite the differences in PSNR. PSNR curves for this image are shown in Fig. 8. The best performing curve is for the regular JPEG approach, followed by the case where the approximated transform was used in both compression and decompression, while the case where there was mismatch is the one where the worst performance is recorded, as expected. For low bit rates, the difference is about $1 \mathrm{~dB}$, which is a good sign that the approximation even in objective terms is not too far off the DCT.

In summary, the conditional transform should be used for higher compression ratios where it makes little difference whether there is a mismatch in the inverse transform. At the same time the transform complexity is nearly halved at only a small decrease in performance, which is typically a few $\mathrm{dB}$ loss in PSNR. Subjectively, the differences among methods are less evident for lower rates.

\section{CONCLUSIONS}

An approximation to the DCT was proposed for use in image compression in order to save computation. They are suitable to JPEG and MPEG in high compression ratio modes, since quantization artifacts might help mask the artifacts caused by the transform approximation. The approximation can typically half the overall computation for low bit rates and where JPEG/MPEG compression of the data blocks is already discarding too much visible information. In other words, savings come from not computing information that is not encoded. The method was shown to be efficient to substitute the DCT in JPEG/MPEG environments for low bit rate targets. The method in many parts can be extended to encompass other transforms such as the wavelet transform used in JPEG 2000 [13], which is a direction for further exploration.

\section{REFERENCES}

[1] R. Buckley, D. Venable and L. McIntyre, "New developments in color facsimile and internet fax," Proc. of ISEST's Fifth Color Imaging Conference, pp. 296-300, Scottsdale, AZ, Nov. 1997.

[2] W. P. Pennebaker and J. L. Mitchell, JPEG: Still Image Compression Standard, Van Nostrand-Reinhold, 1993.

[3] A. M. Tekalp, Digital Video Processing, Upper Saddle River, NJ, Prentice-Hall, 1995.

[4] R. de Queiroz, "Reduced DCT approximations for low bit rate coding," in this Proceedings.

[5] B. Girod and K. W. Stuhlmuller, "A content dependent fast DCT algorithm for low bit-rate video coding," Proc. ICIP, Chicago, IL, Oct. 1998. 


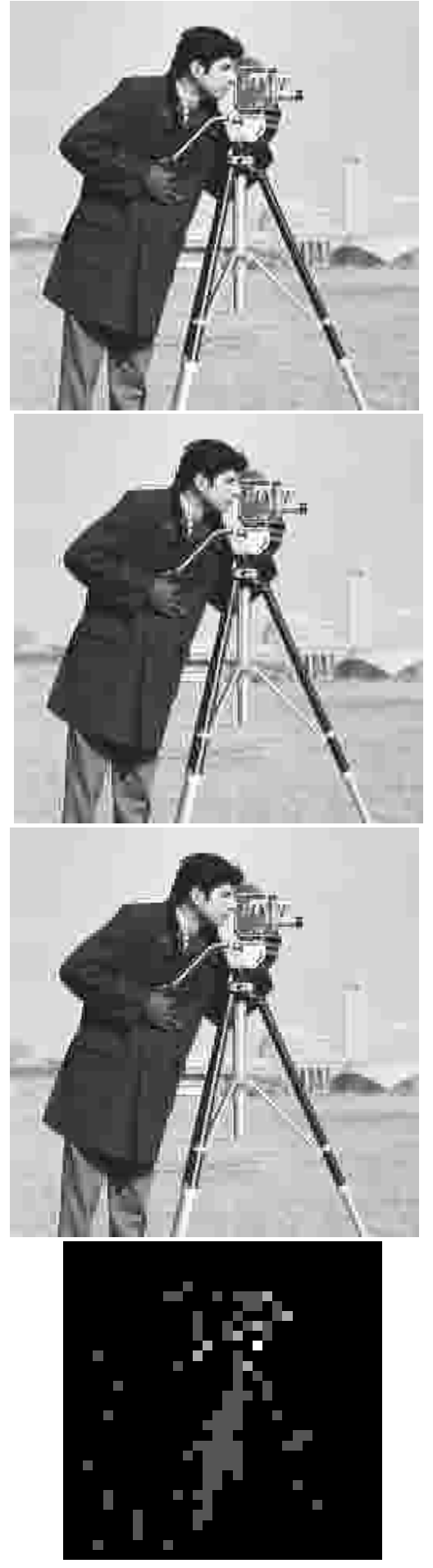

Figure 7. Image cameraman compressed at low bit rates using JPEG. Top left: regular DCT $(0.28$ bpp, PSNR = 24.63dB). Top right: approximated compression but using regular inverse DCT $\mathbf{( 0 . 2 7}$ bpp, PSNR = 23.86dB). Bottom left: approximated transform (0.27 bpp, PSNR $=23.76 \mathrm{~dB})$. Bottom right: map with number of active bands.

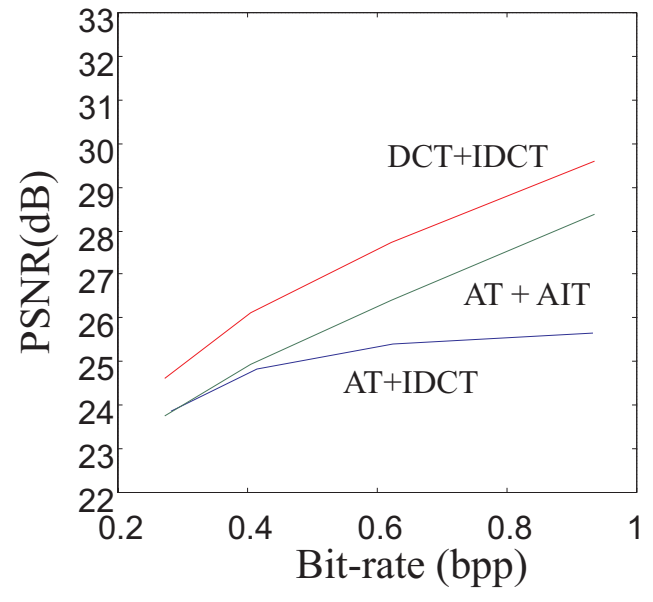

Figure 8. PSNR plots for the conditional transform approach. A(I)T refers to the approximated (inverse) transform.

[6] K. Lengwehasatit and A. Ortega, "DCT computation based on variable complexity fast approximations," Proc. ICIP, Chicago, IL, Oct. 1998.

[7] S.-H. Jung, S. K. Mitra and D. Mukherjee, "Subband DCT : definition, analysis and applications," IEEE Trans. on Circuits and Systems for Video Tech., vol. 6, pp. 273286, June 1996.

[8] I. Richardson and Y. Zhao, "Adaptive algorithms for variable-complexity video coding," Proc. of ICIP, Greece, Oct. 2001.

[9] I-M. Pao and M. T. Sun, "Modelling DCT coefficients for fast video encoding," IEEE Trans. on Circuits and Systems for Video Tech., Vol. 9, pp. 608-616, June 1999.

[10] K. R. Rao and P. Yip Discrete Cosine Transform : Algorithms, Advantages, Applications, San Diego, CA : Academic Press, 1990.

[11] C. Loeffler, A. Ligtenberger, and G. Moschytz, "Practical fast 1-D DCT algorithms with 11 multiplications," Proc. ICASSP, pp. 988-991, 1989.

[12] N. Cho and S. Lee, "Fast algorithm and implementation of the 2D DCT," IEEE Trans. on Circuits and Systems, Vol. 38, pp. 297-305, Mar. 1991.

[13] ISO/IEC JTC1/SC29 WG1, JPEG 2000 Committee, Final Draft Intl. Standard, Sep. 25, 2000. 